Review

\title{
Some Construction Methods of Aggregation Operators in Decision-Making Problems: An Overview
}

\author{
Azadeh Zahedi Khameneh ${ }^{1, *}$ (D) and Adem Kilicman 1,2,* (D) \\ 1 Institute for Mathematical Research, Universiti Putra Malaysia, 43400 UPM Serdang, Selangor, Malaysia \\ 2 Department of Mathematics, Faculty of Science, Universiti Putra Malaysia, \\ 43400 UPM Serdang, Selangor, Malaysia \\ * Correspondence: zk.azadeh@upm.edu.my (A.Z.K.); akilic@upm.edu.my (A.K.)
}

Received: 31 January 2020; Accepted: 29 February 2020; Published: 1 May 2020

\begin{abstract}
Aggregating data is the main line of any discipline dealing with fusion of information from the knowledge-based systems to decision-making. The purpose of aggregation methods is to convert a list of objects, all belonging to a given set, into a single representative object of the same set usually by an $n$-ary function, so-called aggregation operator. As the useful aggregation functions for modeling real-life problems are limited, the basic problem is to construct a proper aggregation operator, usually a symmetric one, for each situation. During the last decades, a number of construction methods for aggregation functions have been developed to build new classes based on the existing well-known operators. There are three main construction methods in common use: transformation, composition, and convex combination. This paper compares these methods with respect to the type of aggregating problems that can be handled by each of them.
\end{abstract}

Keywords: aggregation operators; composite aggregation operators; weighted aggregation operators; transformation; duality; group decision-making

\section{Introduction}

The importance of aggregating in fusion of information, specially in decision-making problems, is, in fact, to get an overview of data for taking the final action. However, there are diverse strategies to reach the aggregated value: aggregation functions are one of the most effective and simple methods in this area. Aggregation functions are a mathematical way to summarize an $n$-tuple of information into a single output by means of non-decreasing functions where the output should remain in the same set as the input one. In literature, the non-decreasing function $A: \bigcup_{n \in \mathbb{N}}[0,1]^{n} \rightarrow[0,1]$ is usually considered as the standard definition of aggregation functions where the non-decreasing property of $A$ shows increasing values of inputs increases the aggregated value. Moreover, the aggregation function $A$ fulfills the boundary conditions $A(0, \cdots, 0)=0$ and $A(1, \cdots, 1)=1$ that guaranty the aggregating of minimal and maximal inputs are respectively minimal and maximal output.

Group decision-making problems that refer to as multi-person or multi-observer decision situations, are one of the main application fields of aggregation functions theory. Such problems usually contain two key phases known as consensus and selection (c.f. [1,2]) where the main objective of consensus is to obtain the consent over different judgments, not necessarily the full agreement of individuals participating in a decision-making problem, whereas selection phase contributes to find the optimum solution (c.f. [3]). According to the final goal in different decision situations, over the past decades a number of methods have been applied to reach consensus in group decision-making problems. The statistical tools and mathematical concepts, such as mean 
value, median, minimum, maximum, triangular norms [4], and fuzzy integrals [5,6] are widely used to aggregating data. On the other hand, dealing with linguistic information and non-numerical variables to express decision-makers' judgments in real-world decision-making problems highlights the use of fuzzy set theory [7] and soft set theory [8] in this area. As a result, most of the aggregating techniques have been adapted to these concepts. For instance, the ordered weighted average (OWA) operator [9] and the ordered weighted geometric (OWG) operator [10] have been developed to intuitionistic fuzzy operators [11,12], interval-valued intuitionistic fuzzy ordered weighted geometric (IVIFOWG) operator [13], intuitionistic trapezoidal fuzzy ordered weighted averaging (ITFOWA) operator [14], intuitionistic fuzzy-induced geometric (IFIGOWA) operators [15], intuionistic fuzzy generalized aggregation operators [16], fuzzy-induced generalized ordered weighted averaging (FI-GOWA) and fuzzy-induced quasi-arithmetic OWA (Quasi-FI-OWA) operators [17], induced generalized intuitionistic fuzzy ordered weighted averaging (I-GIFOWA) operator [18], interval-valued pythagorean trapezoidal fuzzy ordered weighted averaging (IVPTFOWA) operator [19], and $m$-polar fuzzy soft OWA operator [20] by different researchers.

However, the list of aggregation functions is not limited to these single or hybrid operators. In fact, the class of aggregation functions is huge and choosing the right one for a given problem is the main task of decision-makers in any consensus process. For example, averaging aggregation functions are used widely in real-life situations. To find the overall score of a student based on his/her final academic report, we usually use the arithmetic mean value $A M$. However, if different courses have different importance weights, the weighted arithmetic mean WAM, rather than $A M$, should be applied to get the correct result. However, this formula can give rise to misleading result if we want to consider the effect of final exams schedule on the student's performance, where the courses' weights are computed based on the exams timetable rather than how much that course/exam is difficult. Since then, a survey on aggregation functions can give an insight to the experts to enhance their performance for choosing the right one. $\mathrm{Xu}$ and $\mathrm{Da}$ [21] fulfilled the first overview of the aggregation operators in 2003 by reviewing the existing main aggregation operators. The authors of [22-24] focused on properties and classification of aggregation functions. Grabisch et al. [25] made a review only on averaging aggregation operators. Recently, Rosanisah Mohd and Lazim Abdullah [26] provided an overview of different types of aggregation functions used in decision-making from year to year. However, these works did not focus on the type of consensus problem that may handled by different construction methods of aggregation operators.

Finding a proper aggregation operator for the situation with a complex consensus scenario and based on the existing formulas is not always straightforward. We usually need to develop the class of aggregation functions by constructing new ones based on the existing operators. There are a number of techniques to construct different classes of aggregation operators with different properties [23,27-34] where they start from given aggregation functions and continue by constructing new ones. A review paper over such techniques allows us to have an insight of not only the problems that can be solved by them, but also situations that cannot be handled by these methods. To this end, we compare three analytical approaches namely transformation, composition and convex combination, that are used to generate new aggregation functions based on existing ones, to address the following research questions.

1. Which consensus problems can be handled by each of these aggregating techniques?

2. What are the limitations of each technique?

This paper aims to provide an overview of three key construction methods of aggregation functions, namely, transformation, composition, and convex combination, to compare them with respect to type of aggregating problems that can be handled by each of them. This can help researchers gain a more comprehensive understanding of advantages as well as limitations of each constructing method of aggregation functions. We focus on consensus problems that may be solved by these construction techniques of aggregation operators and present a summary of some recent as well as older results. To this end, in Section 2, basic definitions and properties of aggregation functions are recalled. Sections $3-5$ are, respectively, devoted to review of construction methods 
for aggregation functions including transformation of aggregation operator, composite aggregations, and weighted rule of aggregation operators based on convex combination approach. In Section 6, we compare these constructing methods by some numerical example. Finally, a summary is given in the conclusion section.

\section{Basic Definitions and Properties}

In this section, we recall some basic concepts about aggregation functions. Not that, throughout this paper, we use the following notations; $\mathbb{I} \subset \mathbb{R}$ is the closed unit interval $[0,1]$ and $\mathbb{I}^{n}=\left\{\mathbf{x}_{(n)}=\right.$ $\left.\left(x_{1}, \cdots, x_{n}\right): x_{i} \in \mathbb{I}, i=1, \cdots, n\right\}$ denotes the set of all real $n$-dimensional vectors $\mathbf{x}_{(n)}$ whose components are in the interval $\mathbb{I}$. Moreover, we say $\mathbf{x}_{(n)}=\left(x_{1}, \cdots, x_{n}\right) \leq \mathbf{y}_{(n)}=\left(y_{1}, \cdots, y_{n}\right)$ if and only if $x_{i} \leq y_{i}$, for all $i$.

\section{Definition 1.}

- An aggregation function of dimension $n \in \mathbb{N}$ is an n-ary function $A^{(n)}:[0,1]^{n} \rightarrow[0,1]$ satisfying

A1. $\quad A(x)=x$, for $n=1$ and any $x \in[0,1]$

A2. $\quad A^{(n)}\left(x_{1}, \cdots, x_{n}\right) \leq A^{(n)}\left(y_{1}, \ldots, y_{n}\right)$ if $\left(x_{1}, \cdots, x_{n}\right) \leq\left(y_{1}, \cdots, y_{n}\right)$;

A3. $\quad A^{(n)}(0,0, \cdots, 0)=0$ and $A^{(n)}(1,1, \cdots, 1)=1$.

- An extended aggregation function is the function $A: \bigcup_{n \in \mathbb{N}}[0,1]^{n} \rightarrow[0,1]$ whose restriction $\left.A\right|_{\mathbb{I}^{n}}:=A^{(n)}$ to $\mathbb{I}^{n}$ is the n-ary aggregation function $A^{(n)}$ for any $n \in \mathbb{N}$.

Example 1. The operators

1. median Med defined by $\operatorname{Med}\left(x_{1}, \cdots, x_{n}\right)=x_{\frac{n+1}{2}}$ if $n$ is odd and $\operatorname{Med}\left(x_{1}, \cdots, x_{n}\right)=\frac{1}{2}\left[x_{\frac{n}{2}}+x_{\frac{n}{2}+1}\right]$ if $n$ is even where $x_{1} \leq x_{2} \leq \cdots \leq x_{n}$;

2. arithmetic mean $A \bar{M}\left(\mathbf{x}_{(n)}\right)=\frac{1}{n} \sum_{i=1}^{n} x_{i}$;

3. weighted arithmetic mean $\operatorname{WAM}\left(\mathbf{x}_{(n)}\right)=\sum_{i=1}^{n} w_{i} x_{i}$ where $w_{i} \in[0,1]$ and $\sum_{i=1}^{n} w_{i}=1$;

4. geometric mean $\operatorname{GM}\left(\mathbf{x}_{(n)}\right)=\left(\prod_{i=1}^{n} x_{i}\right)^{\frac{1}{n}}$;

5. harmonic mean $\operatorname{HM}\left(\mathbf{x}_{(n)}\right)=\frac{n}{\sum_{i=1}^{n} \frac{1}{x_{i}}}$;

6. $\quad$ minimum $\operatorname{Min}\left(\mathbf{x}_{(n)}\right)=\min _{i=1}^{n} x_{i}$ and maximum $\operatorname{Max}\left(\mathbf{x}_{(n)}\right)=\max _{i=1}^{n} x_{i}$;

7. product function $\Pi\left(\mathbf{x}_{(n)}\right)=\prod_{i=1}^{n} x_{i}$;

8. projection function to the $k$ th coordinate $P_{k}\left(\mathbf{x}_{(n)}\right)=x_{k}$

are several well-known examples of extended aggregation functions.

Remark 1. Note that, we have the weakest aggregation operator $A_{w}$ and the strongest aggregation operator $A_{s}$ defined by

$$
A_{w}=\left\{\begin{array}{ll}
1 & \text { if } x_{1}=x_{2}=\cdots=x_{n}=1 \\
0 & \text { else }
\end{array} \quad A_{s}= \begin{cases}0 & \text { if } x_{1}=x_{2}=\cdots=x_{n}=0 \\
1 & \text { else }\end{cases}\right.
$$

where for any aggregation operator $A$

$$
A_{w} \leq A \leq A_{s}
$$

Definition 2. The aggregation function $A:[0,1]^{n} \rightarrow[0,1]$

- has a neutral element $e \in[0,1]$ if

$$
A\left(x_{1}, \cdots, x_{i-1}, e, x_{i+1}, \cdots, x_{n}\right)=A\left(x_{1}, \cdots, x_{i-1}, x_{i+1}, \cdots, x_{n}\right)
$$

- has an annihilator element (absorbing element or zero element) $a \in[0,1]$ if

$$
A\left(x_{1}, \cdots, x_{i-1}, a, x_{i+1}, \cdots, x_{n}\right)=a
$$


- has no zero divisors if it has the zero element $a$, and

$$
A\left(x_{1}, \cdots, x_{n}\right)=a \Longrightarrow \exists_{1 \leq s \leq n} s: x_{s}=a
$$

Therefore, if an aggregation function has a neutral element $e$, it can be omitted from the initial list of objects without any influence on the final aggregated value. Thus, in a decision-making problem, existence of the neutral element $e$ means that the rest of arguments except $e$ fulfill the aggregation process. If an aggregation function has the annihilator element $a$, then adding it to the list of arguments means that only the argument $a$ fulfills the aggregation stage. In fact, the annihilator element $a$ acts as a veto or qualifying score.

Example 2. The product function $\Pi$ and the minimum function Min have the neutral element 1 , whereas 0 is the neutral element of the maximum function Max. Obviously, 1 is the annihilator for Max and 0 is the annihilator element of $\Pi, M i n$, and GM where the latter has no neutral element. Operators $A_{w}$ and $A_{s}$ are without neutral element, whereas $A_{w}$ has an annihilator $a=0$ and $A_{s}$ has an annihilator $a=1$. The operators $A M$ and $P_{k}$ are examples of aggregation functions without neutral and annihilator elements.

Example 3. Operator $A_{c}: \bigcup_{n \in \mathbb{N}}[0,1]^{n} \rightarrow[0,1]$ defined by

$$
A_{c}=\left(x_{1}, \cdots, x_{n}\right)=\max \left(0, \min \left(1, c+\sum_{i=1}^{n}\left(x_{i}-c\right)\right)\right)
$$

where $c \in[0,1]$ is an aggregation function that has neutral element $e=c$ and without annihilator.

\subsection{Classification of Aggregation Functions}

There are four main classes of aggregation functions, including conjunctive, disjunctive, averaging, and remaining aggregation functions, defined by their relationship to Min and Max functions and proposed in [35] for the first time.

Definition 3. The aggregation function $A:[0,1]^{n} \rightarrow[0,1]$ is called

- Conjunctive if for every $\mathbf{x}_{(n)} \in \mathbb{I}^{n}, A\left(\mathbf{x}_{(n)}\right)$ is bounded by the minimum function:

$$
A\left(\mathbf{x}_{(n)}\right) \leq \operatorname{Min}\left(\mathbf{x}_{(n)}\right)=\min \left(x_{1}, \cdots, x_{n}\right)
$$

- Disjunctive if for every $\mathbf{x}_{(n)} \in \mathbb{I}^{n}, A\left(\mathbf{x}_{(n)}\right)$ is bounded by the maximum function:

$$
A\left(\mathbf{x}_{(n)}\right) \geq \operatorname{Max}\left(\mathbf{x}_{(n)}\right)=\max \left(x_{1}, \cdots, x_{n}\right)
$$

- $\quad$ Average whenever for all $\mathbf{x}_{(n)} \in \mathbb{I}^{n}$ we have:

$$
\operatorname{Min}\left(\mathbf{x}_{(n)}\right) \leq A\left(\mathbf{x}_{(n)}\right) \leq \operatorname{Max}\left(\mathbf{x}_{(n)}\right)
$$

According to [36], if $\mathcal{A}$ is the collection of all aggregation functions, $\mathcal{C}$ shows the class of conjunctive functions, $\mathcal{D}$ is the class of disjunctive functions, $\mathcal{P}$ shows the pure averaging functions, and $\mathcal{R}=$ $\mathcal{A} /(\mathcal{C} \cup \mathcal{D} \cup \mathcal{P})$ is all remaining aggregation functions, then $(\mathcal{C}, \mathcal{D}, \mathcal{P}, \mathcal{R})$ forms a partition on $\mathcal{A}$.

Example 4. Operators $\Pi, A_{w}$, and Min are examples of conjunctive aggregation functions, whereas the operators $\operatorname{PS}\left(x_{1}, x_{2}\right)=x_{1}+x_{2}-x_{1} \cdot x_{2}, A_{s}$ and Max are disjunctive aggregation functions.

Remark 2. Note that if the conjunctive and disjunctive aggregation functions have neutral elements, they are, respectively, $e=1$ and $e=0$. 
From monotonic property of $A$, it is easy to check that the property of averaging is equivalent to the idempotency of aggregation function $A$ where the aggregation function $A:[0,1]^{n} \rightarrow[0,1]$, for $n \in \mathbb{N}$, is called idempotent if $A(x, \cdots, x)=x$ for all $x \in[0,1]$.

If an aggregation operator $A$ has the neutral element $e$, then $A$ is idempotent at $e$. Similarly, if $A$ has the annihilator $a$, then $A$ is idempotent at $a$. Thus, 0 and 1 are idempotent elements for each aggregation operator, called trivial idempotent elements.

Example 5. The arithmetic mean AM, the geometric mean GM, the operator Min, the operator Max, and the projection operator $P_{k}$ are average (idempotent) aggregation functions. $A_{c}$ is not an average (idempotent) aggregation operator, but has the idempotent elements $0,1, c$ where $c \in[0,1]$.

Remark 3. In multi-criteria decision-making problems, idempotency (averaging) means if all criteria (or decision-makers) are satisfied at the same degree like $x$, then the global score should be $x$.

\section{Triangular Norms and Conorms}

Triangular norms and conorms, or $t$-norms and $t$-conorms in brief, are well-known examples of conjunctive and disjunctive aggregation operators with the neutral elements $e=1$ and $e=0$, respectively, that are associative and commutative.

Definition 4. ([4]) A binary operation $T:[0,1]^{2} \rightarrow[0,1]$ is called a triangular norm or $t$-norm if it is symmetric, associative, non-decreasing function and satisfies the boundary condition $T(x, 1)=x, \forall x \in[0,1]$. A binary operation $S:[0,1]^{2} \rightarrow[0,1]$ is called a triangular conorm or $t$-conorm if it is symmetric, associative, non-decreasing function and satisfies the boundary condition $S(x, 0)=x, \forall x \in[0,1]$.

Example 6. $T_{D}\left(x_{1}, x_{2}\right)=x_{1}$ or $x_{2}$ if $x_{2}=1$ or $x_{1}=1$ and otherwise is zero, $T_{M}\left(x_{1}, x_{2}\right)=\min \left(x_{1}, x_{2}\right)$, $T_{P}\left(x_{1}, x_{2}\right)=x_{1} x_{2}$ and $T_{L}\left(x_{1}, x_{2}\right)=\max \left(x_{1}+x_{2}-1,0\right)$ are some examples for $t$-norms where

$$
T_{D} \leq T_{L} \leq T_{P} \leq T_{M}
$$

On the other hand, operators $S_{D}\left(x_{1}, x_{2}\right)=x_{1}$ or $x_{2}$ if $x_{2}=0$ or $x_{1}=0$ and otherwise is one, $S_{M}\left(x_{1}, x_{2}\right)=$ $\max \left(x_{1}, x_{2}\right), S_{P}\left(x_{1}, x_{2}\right)=x_{1}+x_{2}-x_{1} x_{2}$ and $S_{L}\left(x_{1}, x_{2}\right)=\min \left(x_{1}+x_{2}, 1\right)$ are $t$-conorms such that

$$
S_{M} \leq S_{P} \leq S_{L} \leq S_{D}
$$

\subsection{Properties of Aggregation Functions}

The algebraic and analytic properties of an arbitrary $n$-ary real functions, such as continuity and associativity can be naturally defined for the aggregation function $A:[0,1]^{n} \rightarrow[0,1]$.

Definition 5. The aggregation function $A:[0,1]^{n} \rightarrow[0,1]$, for $n \in \mathbb{N}$, is called

1. Additive if $A\left(\mathbf{x}_{(n)}+\mathbf{y}_{(n)}\right)=A\left(\mathbf{x}_{(n)}\right)+A\left(\mathbf{y}_{(n)}\right)$ for all $\mathbf{x}_{(n)}, \mathbf{y}_{(n)} \in \mathbb{I}^{n}$ such that $\mathbf{x}_{(n)}+\mathbf{y}_{(n)} \in \mathbb{I}^{n}$;

2. Idempotent if $A(x, \cdots, x)=x$ for all $x \in[0,1]$;

3. Symmetric if $A\left(x_{1}, \cdots, x_{n}\right)=A\left(x_{\sigma(1)}, \cdots, x_{\sigma(n)}\right)$ for all $\mathbf{x}_{(n)}=\left(x_{1}, \cdots, x_{n}\right) \in \mathbb{I}^{n}$ where $\sigma$ is any permutation of $\{1, \cdots, n\}$;

4. Bisymmetric if for all $x_{i j} \in[0,1]$ where $i, j \in\{1, \cdots, n\}$ we have $A\left(A\left(x_{11}, \cdots, x_{1 n}\right), \cdots, A\left(x_{n 1}, \cdots, x_{n n}\right)\right)=A\left(A\left(x_{11}, \cdots, x_{n 1}\right), \cdots, A\left(x_{1 n}, \cdots, x_{n n}\right)\right)$;

5. Strongly bisymmetric if $A\left(\mathbf{x}_{(n)}\right)=\mathbf{x}_{(n)}$ for all $\mathbf{x}_{(n)} \in$ I $\mathbb{I}^{n}$ and $A\left(A\left(x_{11}, \cdots, x_{1 n}\right), \cdots, A\left(x_{m 1}, \cdots, x_{m n}\right)\right)=A\left(A\left(x_{11}, \cdots, x_{m 1}\right), \cdots, A\left(x_{1 n}, \cdots, x_{m n}\right)\right)$ for any $m, n \in \mathbb{N}$;

6. Associative if for all $\left(x_{1}, x_{2}, x_{3}\right) \in \mathbb{I}^{3}$ we have $A\left(A\left(x_{1}, x_{2}\right), x_{3}\right)=A\left(x_{1}, A\left(x_{2}, x_{3}\right)\right)$;

7. Continuous if for any $\mathbf{x}_{(n)} \in \mathbb{I}^{n}$ and $\left(x_{i_{j}}\right)_{j \in \mathbb{N}} \in \mathbb{I}^{\mathbb{N}}$ where $i \in\{1, \cdots, n\}$, if $\lim _{j \rightarrow \infty} x_{i_{j}}=x_{i}$ then $\lim _{j \rightarrow \infty} A\left(x_{1_{j}}, \cdots, x_{n_{j}}\right)=A\left(x_{1}, \cdots, x_{n}\right)$ or equivalently $\forall \epsilon>0, \exists \delta>0:$ if $\left|x_{i}-y_{i}\right|<\delta$ where $i \in\{1, \cdots, n\}$ then $\left|A\left(x_{1}, \cdots, x_{n}\right)-A\left(y_{1}, \cdots, y_{n}\right)\right|<\epsilon$; 
8. c-Lipschitz with respect to the norm $\|\|:. \mathbb{R} \rightarrow[0,+\infty)$, if for some constant $c \in(0,+\infty)$ we have: $\left|A\left(\mathbf{x}_{(n)}\right)-A\left(\mathbf{y}_{(n)}\right)\right| \leq c\left\|\mathbf{x}_{(n)}-\mathbf{y}_{(n)}\right\|$ for all $\mathbf{x}_{(n)}, \mathbf{y}_{(n)} \in \mathbb{I}^{n}$.

Example 7. It is evident that the arithmetic mean $A M$ is an example of an additive function. Operator $\Pi$ is not idempotent. Operators $A M, \prod, M i n, M a x, P_{k}$, and $A_{c}$ are continuous aggregation operators, which all also fulfill the 1-Lipschitz property. Operator $A M$ is the only aggregation function that is $\frac{1}{n}$-Lipschitz for all $n \in \mathbb{N}$ :

$$
1=|A M(1, \cdots, 1)-A M(0, \cdots, 0)|=\frac{1}{n} \sum_{i=1}^{n}|1-0|
$$

whereas GM is an example of a continuous aggregation operator which is not Lipschitz. $A_{w}$ and $A_{s}$ are examples of non-continuous operators.

Remark 4. Note that the symmetric property of an aggregation function reflects the same importance of single criteria in a multi-criteria decision-making problem, i.e., knowledge of the order of input score is irrelevant.

Moreover, the bisymmetry property of an aggregation operator allows us to obtain an overall score of each candidate according to $n$ judges from different decision-makers based on $m$ criteria by any two following ways. We can first aggregate the numerical scores of each candidate over all criteria given by each decision-maker and then aggregate these values, or we may first aggregate the scores of the candidate on the basis of each criteria and then merge them over all decision-makers.

Example 8. $A M, G M, \prod, M e d, M i n, M a x, O W A$ and $O W G$ are examples of symmetric aggregation functions, whereas $P_{k}$ and WAM are non-symmetric aggregation functions.

Remark 5. The associativity property of an aggregation function allows us to start with aggregation procedure before knowing all inputs to be aggregated. Then, additional input can be simply aggregated with the output of the previous aggregating step.

Example 9. Aggregation operators $A_{w}, A_{s}, \operatorname{Min}, \operatorname{Max}, \Pi$, and $P_{k}$ are examples of associative functions. Operators $A_{c}$ and GM are non-associative aggregation operators.

\subsection{Construction Methods of Aggregation Functions}

In aggregation operators theory, construction methods are one of the important issues that should be addressed. There exist a large number of aggregation operators, some simple and straightforward and some very complex, with different properties. However, finding a proper one for a specific situation may be difficult. Sometimes the traditional operators are not suitable and experts try to develop new aggregation functions from the existing ones. In this case, the generic problem that should be solved is below.

Problem I. Constructing an aggregation function $A \in \mathcal{A}$, possibly with some additional properties, to find the best output.

To cope with Problem I, several construction methods have been discussed in literature to create new aggregation functions. In the following sections, we review three commonly used construction methods of aggregation functions, namely transformation, composition, and convex combination, which answer Problem I. We also compare the type of consensus problems that can be solved by each of them.

\section{Transformation of Aggregation Functions}

The idea of transformation of functions can be used to construct new aggregation functions that inherit the algebraic and topological properties of the original aggregation functions. 
Proposition 1. Let $A: \mathbb{I}^{n} \rightarrow \mathbb{I}$ be an n-ary aggregation function and $\phi: \mathbb{I} \rightarrow \mathbb{I}$ be a monotone bijection where $\mathbb{I}, \mathbb{J}$ are real intervals. Then, $A_{\phi}: \mathbb{J}^{n} \rightarrow \mathbb{J}$ given by

$$
A_{\phi}\left(x_{1}, \cdots, x_{n}\right)=\phi^{-1}\left(A\left(\phi\left(x_{1}\right), \cdots, \phi\left(x_{n}\right)\right)\right)
$$

is an n-ary aggregation function on $\mathbb{J}^{n}$.

However, the analytical properties of $A$ such as Lipschitz property, additivity or linearity are not be inherited by $A_{\phi}$.

Observe that the transformation formula can be successfully applied to change the scale from example $\mathbb{I}^{n}$ into the $\mathbb{J}^{n}$ by means of a monotone bijection $\phi: \mathbb{J} \rightarrow \mathbb{I}$ where $\mathbb{J}$ is any real interval.

If $A$ has the neutral element $e$, then by applying transformation formula on scales $[0, e]$ and $[e, 1]$ we can get two new aggregation operators based on $A$ and with the neutral elements 1 and 0 , respectively. For example, let $A: \bigcup_{n \in \mathbb{N}}[0,1]^{n} \rightarrow[0,1]$ be an aggregation function defined by

$$
A\left(x_{1}, \cdots, x_{n}\right)=\frac{\prod_{i=1}^{n} x_{i}}{\prod_{i=1}^{n} x_{i}+\prod_{i=1}^{n}\left(1-x_{i}\right)}
$$

with $\frac{0}{0}=0$, that is an associative and symmetric aggregation function with neutral element $e=0.5$. Then, by assumption $\phi_{0}:[0,1] \rightarrow[0,0.5]$ where $\phi_{0}(x)=\frac{1}{2} x$ we can define $A_{[0]}: \bigcup_{n \in \mathbb{N}}[0,1]^{n} \rightarrow[0,1]$ by

$$
A_{[0]}\left(x_{1}, \cdots, x_{n}\right)=\frac{2 \prod_{i=1}^{n} x_{i}}{\prod_{i=1}^{n} x_{i}+\prod_{i=1}^{n}\left(2-x_{i}\right)}
$$

that is an associative and symmetric aggregation function with neutral element $e=1$. Moreover, if we put $\phi_{1}:[0,1] \rightarrow[0.5,1]$ where $\phi_{1}(x)=\frac{x+1}{2}$ we can define $A_{[1]}: \bigcup_{n \in \mathbb{N}}[0,1]^{n} \rightarrow[0,1]$ by

$$
A_{[1]}\left(x_{1}, \cdots, x_{n}\right)=\frac{2 \prod_{i=1}^{n}\left(x_{i}+1\right)}{\prod_{i=1}^{n}\left(x_{i}+1\right)+\prod_{i=1}^{n}\left(1-x_{i}\right)}-1
$$

that is an associative and symmetric aggregation function with neutral element $e=0$. Similarly, if $A$ has the annihilator $a$, then by applying transformation formula on scales $[0, a]$ and $[a, 1]$, we can get two aggregation operators with annihilators 1 and 0 , respectively.

\subsection{Duality of Aggregation Functions}

One of the most applied transformations is the duality transformation where $\phi(x)=1-x$. Applying this transformation to any aggregation operator $A$, the dual aggregation function $A^{d}$ of $A$ is obtained. Indeed, duality is one of the simplest methods to develop an aggregation function on the basis of a given one.

For any (extended) aggregation function $A$, the mapping $A^{d}: \bigcup_{n \in \mathbb{N}}[0,1]^{n} \rightarrow[0,1]$ defined by

$$
A^{d}\left(x_{1}, \cdots, x_{n}\right)=1-A\left(1-x_{1}, \cdots, 1-x_{n}\right)
$$

is called the dual aggregation function of $A$.

Clearly, the minimum and the maximum functions are dual of each other. The operators $A_{w}$ and $A_{s}$ are also dual of each other. Moreover, if the product function $\Pi\left(\mathbf{x}_{(n)}\right)=\prod_{i=1}^{n} x_{i}$ is given, then its dual, i.e., the probabilistic sum $P S\left(\mathbf{x}_{(n)}\right)=1-\prod_{i=1}^{n}\left(1-x_{i}\right)$, is also an aggregation function.

Remark 6. The aggregation operators $A$ and $A^{d}$ have the same analytical and algebraic properties, e.g., if $A$ is continuous or symmetric then $A^{d}$ is also continuous or symmetric.

As the operators Min, Max are dual of each other, we clearly have the following result. 
Proposition 2. Let $A: \bigcup_{n \in \mathbb{N}}[0,1]^{n} \rightarrow[0,1]$ be an aggregation operator and $A^{d}$ be its dual.

- If $A$ is average (or alternatively idempotent), then $A^{d}$ is also average (idempotent).

- If $A$ is conjunctive (disjunctive), then $A^{d}$ is disjunctive (conjunctive).

Remark 7. If $A$ has the neutral element $e$, then $1-e$ is the neutral element of $A^{d}$. Moreover, if $a$ is the annihilator of $A$, then $A^{d}$ has the annihilator element $1-a$.

The aggregation operator $A$ is called self-dual (or symmetric sum) if and only if $A^{d}=A$ or equivalently for any $\mathbf{x}_{(n)} \in \mathbb{I}^{n}: A\left(\mathbf{1}-\mathbf{x}_{(n)}\right)=1-A\left(\mathbf{x}_{(n)}\right)$. The arithmetic mean $A M$ is a well-known example for self-dual aggregation functions, as $A M^{d}\left(x_{1}, \cdots, x_{n}\right)=1-\frac{n-\sum_{i=1}^{n} x_{i}}{n}=\frac{\sum_{i=1}^{n} x_{i}}{n}$. The median operator Med and the weighted arithmetic mean WAM are also self-dual aggregation functions.

However, in general case, the most aggregation functions are not self-dual. However, there is a technique (c.f. [28], Propositions 6 and 8) to make a self-dual function by using the average of aggregation function $A$ and its dual, i.e., $A^{d}$.

Proposition 3. ([28]) An aggregation operator $A: \bigcup_{n \in \mathbb{N}}[0,1]^{n} \rightarrow[0,1]$ is self-dual if and only if there exists an aggregation operator $B$ such that $A=\hat{B}$ where

$$
\hat{B}\left(x_{1}, \cdots, x_{n}\right)=\frac{B\left(x_{1}, \cdots, x_{n}\right)+B^{d}\left(x_{1}, \cdots, x_{n}\right)}{2}
$$

and it is called the core of aggregation operator $B$.

According to Proposition 3, by having an aggregation function $B$, we can generate a self-dual aggregation function $A$. For example, the arithmetic mean of Min and Max, i.e.,

$$
A\left(\mathbf{x}_{(n)}\right)=\frac{\min \left(\mathbf{x}_{(n)}\right)+\max \left(\mathbf{x}_{(n)}\right)}{2}
$$

or the arithmetic mean of product function and probabilistic sum, i.e.,

$$
A\left(\mathbf{x}_{(n)}\right)=\frac{1+\prod_{i=1}^{n} x_{i}-\prod_{i=1}^{n}\left(1-x_{i}\right)}{2}
$$

where the latter is mean value for $n=2$, are two new aggregation operators that are self-dual functions.

Remark 8. Triangular norms and their dual, i.e., the class of triangular conorms, are the most important and useful examples for the concept of duality in aggregation theory. Furthermore, as $t$-norms and $t$-conorms are dual to each other, by condition $T\left(x_{1}, x_{2}\right)=1-S\left(1-x_{1}, 1-x_{2}\right)$ it will be sufficient to deal with $t$-norms only and accordingly the properties of t-conorms are obtained.

\subsection{Quasi-Arithmetic Means}

One of the most important classes of aggregation functions generated by the transformation formula is the quasi-arithmetic means as the transformation of $A M$ [29]. If the arithmetic mean $A M$ defined by $\operatorname{AM}\left(x_{1}, \cdots, x_{n}\right)=\frac{1}{n} \sum_{i=1}^{n} x_{i}$ is given and $f:[0,1] \rightarrow \mathbb{R}$ is a continuous strictly monotone function, then by (1) we can get the aggregation function $A M_{f}: \bigcup_{n \in \mathbb{N}}[0,1]^{n} \rightarrow[0,1]$ defined by

$$
A M_{f}\left(x_{1}, \cdots, x_{n}\right)=f^{-1}\left(A M\left(f\left(x_{1}\right), \cdots, f\left(x_{n}\right)\right)\right)=f^{-1}\left(\frac{1}{n} \sum_{i=1}^{n} f\left(x_{i}\right)\right)
$$

that is called quasi-arithmetic mean. The function $f$ defined in (4) is called a generator of $A M_{f}$. 
The quasi-arithmetic means are averaging aggregation functions that can be considered as the modern definition of mean value in the sense of Cauchy [37]. Therefore, they may be viewed as the solution for the following problem.

Problem II. Constructing an averaging aggregation function $A \in \mathcal{A}$ such that Min $\leq A \leq \operatorname{Max}$.

Clearly, the class of quasi-arithmetic means includes the most commonly useful averaging aggregation functions, i.e., the arithmetic mean and the geometric mean. The below example provides some well-known instances of quasi-arithmetic means generated by means of different formulas for $f$.

Example 10. In Equation (4),

1. if $f(x)=x$ then $A M_{f}\left(\mathbf{x}_{(n)}\right)=A M\left(\mathbf{x}_{(n)}\right)=\frac{1}{n} \sum_{i=1}^{n} x_{i}$ (arithmetic mean),

2. if $f(x)=x^{2}$ then $A M_{f}\left(\mathbf{x}_{(n)}\right)=Q M\left(\mathbf{x}_{(n)}\right)=\left(\frac{1}{n} \sum_{i=1}^{n} x_{i}^{2}\right)^{1 / 2}$ (quadratic mean mean),

3. if $f(x)=\log x$ then $A M_{f}\left(\mathbf{x}_{(n)}\right)=G M\left(\mathbf{x}_{(n)}\right)=\left(\prod_{i=1}^{n} x_{i}\right)^{1 / n}$ (geometric mean),

4. if $f(x)=\frac{1}{x}$ then $A M_{f}\left(\mathbf{x}_{(n)}\right)=H M\left(\mathbf{x}_{(n)}\right)=\frac{1}{\frac{1}{n} \sum_{i=1}^{n} \frac{1}{x_{i}}}$ (harmonic mean),

5. if $f(x)=e^{\alpha x}$ where $0 \neq \alpha \in \mathbb{R}$ then $A M_{f}\left(\mathbf{x}_{(n)}\right)=E M_{\alpha}\left(\mathbf{x}_{(n)}\right)=\frac{1}{\alpha} \ln \left(\frac{1}{n} \sum_{i=1}^{n} e^{\alpha x_{i}}\right)$ (exponential mean).

The following result shows the relation between quasi-arithmetic means generated by two functions $f$ and $g$.

Theorem 1. ([29]) Let $f, g:[0,1] \rightarrow \mathbb{R}$ be continuous and strictly monotonic functions where $g$ is also increasing. Then,

1. $A M_{f} \leq A M_{g}$ if and only if $g \circ f^{-1}$ is convex.

2. $A M_{f}=A M_{g}$ if and only if $g \circ f^{-1}$ is linear, i.e., $g(x)=a f(x)+b$ where $a, b \in \mathbb{R}$ and $a \neq 0$.

Moreover, the next theorem talks about an axiomatization of quasi-arithmetic mean as an $n$-ary aggregation function.

Theorem 2. ([25]) The function $F:[0,1]^{n} \rightarrow \mathbb{R}$ is symmetric, continuous, strictly increasing, idempotent, and bisymmetric if and only if there is a continuous and strictly monotonic function $f:[0,1] \rightarrow \mathbb{R}$ such that $F:=A M_{f}$ is the quasi-arithmetic mean generated by $f$.

\section{Composite Aggregation Functions}

Another class of constructing methods to aggregation operators is composition over some given aggregation functions. The main objective for applying the composition technique to aggregation functions is to give an alternative aggregating approach when we face multi-source data. Therefore, such technique can be addressed the following problem.

Problem III. Constructing an aggregation function $A \in \mathcal{A}$ to combine the input data that have been merged by different techniques.

Proposition 4. ([23]) Let $B:[0,1]^{m} \rightarrow[0,1]$ and $A_{i}:[0,1]^{n} \rightarrow[0,1]$ for $i \in\{1, \cdots, m\}$ are some aggregation functions where $m, n \in \mathbb{N} /\{1\}$. Then, the composite function $C_{B ; A_{1}, \cdots, A_{m}}:[0,1]^{n} \rightarrow[0,1]$ defined by

$$
C_{B ; A_{1}, \cdots, A_{m}}\left(x_{1}, \cdots, x_{n}\right)=B\left(A_{1}\left(x_{1}, \cdots, x_{n}\right), \cdots, A_{m}\left(x_{1}, \cdots, x_{n}\right)\right)
$$

is an aggregation function if and only if $B$ is an idempotent (averaging) aggregation operator.

Note that in Equation (5), each $x_{i}$ is aggregated by each of the inner aggregation functions $A_{i}$, so totally $m$-times. 
Remark 9. In reality, Equation (5) can be seen as a decision-making situation involving $m$ decision-makers where each $A_{i}$ correspond to the ith expert and operator $B$ to the head or mentor of the group.

For example, if we take $B$ in (5) as the weighted arithmetic mean WAM with weighting vector $\mathbf{w}_{(m)}=\left(w_{1}, \cdots, w_{m}\right)$, then the function $C_{B ; A_{1}, \cdots, A_{m}}:=\sum_{i=1}^{m} w_{i} A_{i}$ is the convex combination of aggregation functions $A_{1}, \cdots, A_{m}$. As another simple example if $B:=\max$ or $B:=\min$, then $C_{B ; A_{1}, \cdots, A_{m}}:=\max \left(A_{1}, \cdots, A_{m}\right)$ and $C_{B ; A_{1}, \cdots, A_{m}}:=\min \left(A_{1}, \cdots, A_{m}\right)$, respectively.

\subsection{Composition over Different Source of Data}

To aggregate a list of data coming from two or more different sources into a single output by using different aggregation functions that are defined according to the type of each source of input data, an alternative construction method is proposed in [27] to solve the following problem.

Problem IV. Constructing an aggregation function $A \in \mathcal{A}$ to combine two different types of input data.

Proposition 5. ([27]) Let $B:[0,1]^{2} \rightarrow[0,1], A_{1}:[0,1]^{n} \rightarrow[0,1]$ and $A_{2}:[0,1]^{m} \rightarrow[0,1]$ be some aggregation functions where $m, n \in \mathbb{N} /\{1\}$. Then, the composite function $D_{B ; A_{1}, A_{2}}:[0,1]^{n+m} \rightarrow[0,1]$ given by

$$
D_{B ; A_{1}, A_{2}}\left(x_{1}, \cdots, x_{n+m}\right)=B\left(A_{1}\left(x_{1}, \cdots, x_{n}\right), A_{2}\left(x_{n+1}, \cdots, x_{n+m}\right)\right)
$$

is an aggregation operator that is called double aggregation function.

For example, in Proposition 5, let $B$ be the median operator Med. Then, the function $D_{B ; A_{1}, A_{2}}$ given by (6) is the average value of aggregation functions $A_{1}$ and $A_{2}$, i.e., $D_{B ; A_{1}, A_{2}}:=\frac{A_{1}+A_{2}}{2}$ is the arithmetic mean of functions $A_{1}$ and $A_{2}$.

Remark 10. The main difference between aggregation functions given in (5) and (6) is in the domains of inner aggregation functions. In (6), each input $x_{j}$ from the initial list of data is aggregated by only one of the inner aggregation functions $A_{i}$. However, in (5), each input $x_{j}$ is aggregated by each of the inner aggregation functions $A_{i}$. Thus, Proposition 5 allows us to aggregate a list of data coming from two or more different sources into a single output based on different aggregation functions that are defined according to each source of data.

The Proposition 5 can be generalized for aggregation of $m$ lists of inputs $\mathbf{x}_{\mathbf{1}}, \cdots, \mathbf{x}_{\mathbf{m}}$ by an aggregation function $D_{B ; A_{1}, \cdots, A_{m}}:[0,1]^{n} \rightarrow[0,1]$ given by $D_{B ; A_{1}, \cdots, A_{m}}\left(\mathbf{x}_{\mathbf{1}}, \cdots, \mathbf{x}_{\mathbf{m}}\right)=$ $B\left(A_{1}\left(\mathbf{x}_{\mathbf{1}}\right), \cdots, A_{m}\left(\mathbf{x}_{\mathbf{m}}\right)\right)$ where for each $i \in\{1, \cdots, m\}: \mathbf{x}_{\mathbf{i}} \in[0,1]^{n_{i}}, \sum_{i=1}^{m} n_{i}=n$ and the outer and inner aggregation functions are defined by mappings $B:[0,1]^{m} \rightarrow[0,1]$ and $A_{i}:[0,1]^{n_{i}} \rightarrow[0,1]$.

\subsection{Composition over Sub-Groups of Data}

An interesting composition method of aggregation functions was recently proposed in [38]. This method can help handle the problem of partial agreement (not necessarily the full agreement) when $\alpha$ number of total $n$ criteria or decision-makers are sufficient to reach consensus. The aim of this method is to overcome the following difficulty.

Problem V. Constructing an alternative aggregating approach in multi-criteria decision-making problems with $n$ arguments where any possible list of $\alpha \leq n$ arguments (not necessarily all $n$ arguments) can affect the final decision at the consensus level $\alpha$.

Before giving the explanation of this method, first, let $X=\left\{x_{1}, \cdots, x_{n}\right\}$ be the universal set of $n$ elements and $C_{n, \alpha}$ stands for $\alpha$-combination operator of $n$ where $C_{n, \alpha}^{i}\left\{x_{1}, \cdots, x_{n}\right\}$ represents the $i$ th $\alpha$-combination from the set $X$. We apply the operator $C_{n, \alpha}$ for the index set $I=\{1, \cdots, n\}$ to cut-off $I$ into the different subsets $I_{i} \subseteq I$ with cardinality $\left|I_{i}\right|=\alpha$ such that $C_{n, \alpha}(I)=\left\{I_{1}, \cdots, I_{k}\right\}$, where for $i=1, \cdots, k$ : $I_{i}$ shows the $i$ th $\alpha$-combination of $I$. By using the permutation operator 
$\sigma^{*}:\{1, \cdots, k\} \rightarrow\{1, \cdots, k\}$, we rearrange the collection $C_{n, \alpha}(I)$ to provide a lexicographical order on $C_{n, \alpha}(I)$.

For any extended aggregation functions $A, B$ we can define an aggregation function $F_{\alpha ; B, A}$ : $\bigcup_{n \in \mathbb{N}}[0,1]^{n} \rightarrow[0,1]$ by the following theorem.

Theorem 3. ([38]) Let $A, B: \bigcup_{n \in \mathbb{N}}[0,1]^{n} \rightarrow[0,1]$ be two (extended) aggregation functions. The function $F_{\alpha ; B, A}: \bigcup_{n \in \mathbb{N}}[0,1]^{n} \rightarrow[0,1]$ given by

$$
F_{\alpha ; B, A}^{(n)}\left(x_{1}, \cdots, x_{n}\right)=B^{(k)}\left(A^{(\alpha)}\left(C_{n, \alpha}^{\sigma^{*}(1)}\left\{x_{1}, \cdots, x_{n}\right\}\right), \cdots, A^{(\alpha)}\left(C_{n, \alpha}^{\sigma^{*}(k)}\left\{x_{1}, \cdots, x_{n}\right\}\right)\right): n \in \mathbb{N}
$$

is an (extended) aggregation operator called combination operator-based aggregation function of degree $\alpha$ where $\alpha \in\{1,2, \cdots, n\}$ and $k=\frac{n !}{\alpha !(n-\alpha) !}$ is the binomial coefficient.

In Theorem 3, for any $1 \leq i \leq k$ we have the sequence $C_{n, \alpha}^{\sigma^{*}(i)}\left\{x_{1}, \cdots, x_{n}\right\}=\left(x_{\iota_{i, \sigma(1)}}, \cdots, x_{\iota_{i, \sigma(\alpha)}}\right)$ that traverses the $i$ th $\alpha$-combination of the set $\left\{x_{1}, \cdots, x_{n}\right\}$ in the lexicographical order of $C_{n, \alpha}(I)$ such that for any $j, \iota_{i, \sigma(j)}$ is strictly smaller than $\iota_{i, \sigma(j+1)}$.

Thus, we first apply the operator $A$ for aggregating any $\alpha$-element selection of the set $\left\{x_{1}, \cdots, x_{n}\right\}$, and then use the operator $B$ to combine these obtained values into an unique output.

Example 11. Take the aggregation functions $B:=\max$ and $A:=\min$. If $n=4$ and $\alpha=3$, then the aggregation operator $F_{3 ; \text { max,min }}^{(4)}:[0,1]^{4} \rightarrow[0,1]$, defined by

$$
F_{3 ; \max , \min }^{(4)}\left(x_{1}, x_{2}, x_{3}, x_{4}\right)=\max \left(\min \left(x_{1}, x_{2}, x_{3}\right), \min \left(x_{1}, x_{2}, x_{4}\right), \min \left(x_{1}, x_{3}, x_{4}\right), \min \left(x_{2}, x_{3}, x_{4}\right)\right)
$$

first combines pessimistically any 3-member selection over the alternatives $x_{1}, x_{2}, x_{3}$ and $x_{4}$ and then merges them optimistically to get the overall result.

By changing the aggregation operators $A, B$, we can get different classes of aggregation operator $F_{\alpha ; B, A}^{(n)}$. For example, let $n=3$ and $\alpha=2$. If $B:=\max$ and $A:=\min$, then

$$
F_{2 ; \max , \min }^{(3)}\left(x_{1}, x_{2}, x_{3}\right)=\max \left(\min \left(x_{1}, x_{2}\right), \min \left(x_{1}, x_{3}\right), \min \left(x_{2}, x_{3}\right)\right)=\operatorname{Med}\left(x_{1}, x_{2}, x_{3}\right)
$$

If $A=B:=A M$, then

$$
F_{2 ; A M, A M}^{(3)}\left(x_{1}, x_{2}, x_{3}\right)=\frac{1}{3}\left(\frac{x_{1}+x_{2}}{2}+\frac{x_{1}+x_{3}}{2}+\frac{x_{2}+x_{3}}{2}\right)=\frac{\sum_{i=1}^{3} x_{i}}{3}=A M\left(x_{1}, x_{2}, x_{3}\right)
$$

If $A=B:=\Pi$, then

$$
F_{2 ; \Pi, \Pi}^{(3)}\left(x_{1}, x_{2}, x_{3}\right)=\left(x_{1} x_{2}\right) \cdot\left(x_{1} x_{2}\right) \cdot\left(x_{2} x_{3}\right)=\Pi_{i=1}^{3} x_{i}^{2}
$$

That are not, of course, new operators.

\section{Aggregating of Weighted Input}

An important factor to handle aggregating problem of input data with different importance is weights. Indeed, the correspondence weighting vector $\mathbf{w}_{(n)}=\left(w_{1}, \cdots, w_{n}\right)$ to such input values $x_{1}, \cdots, x_{n}$ can be understood as the vector of cardinality of each input $x_{i}$ where $w_{i}>0$ for $i=1, \cdots, n$. If $\sum_{i=1}^{n} w_{i}=1$, then $\mathbf{w}_{(n)}$ is called the normal weighting vector.

Thus, the aggregation function over weighted data contributes to solve the following problem. 
Problem VI. Constructing an aggregation operators $A_{\mathrm{w}} \in \mathcal{A}$ that permit to consider different weights of the sources or data where in fact,

$$
A_{\mathbf{w}}\left(x_{1}, \cdots, x_{n}\right)=A(\underbrace{x_{1}, \cdots, x_{1}}_{w_{1}}, \cdots, \underbrace{x_{n}, \cdots, x_{n}}_{w_{n}})
$$

To aggregate a list of weighted input values, there are two main methods: (1) using the weighted aggregation operators defined naturally based on the weighting vector, such as weighted arithmetic mean, or (2) applying some techniques like weighted quasi-arithmetic means and weighted rule proposed based on a convex combination in [39] to produce a weighted aggregation operator based on unweighted one.

However, in both methodologies the identification of weights is an interesting topic. In practice, there is no unique strategy to find the associated weighting vector $\mathbf{w}_{(n)}$. Sometimes, these weights are given by the decision-makers or mentors involved in the decision-making problem based on their knowledge, information, and past experiences. The weighting vector can be also determined by a fuzzy linguistic quantifier function $Q:[0,1] \rightarrow[0,1]$ based on the formulation $w_{i}=Q\left(\frac{i}{n}\right)-Q\left(\frac{i-1}{n}\right)$ for all $i=1, \cdots, n$, where the definition of $Q$ may be changed from one case to another one $[9,10,40-43]$. For example, the case " $\mathrm{Q}:=$ most", where "most" is interpreted as $60 \%$ of all data, may be defined by

$$
Q_{\text {most }}(z)= \begin{cases}0 & \text { if } z \leq 0.2 \\ \frac{z-0.2}{0.4} & \text { if } 0.2<z<0.6 \\ 1 & \text { if } z \geq 0.6\end{cases}
$$

that means if at least $60 \%$ of some elements satisfy a property, then most of them certainly (to degree 1 ) satisfy it, when less than $20 \%$ of them satisfy it, then most of them certainly do not satisfy it (satisfy to degree 0 ). If between $20 \%$ and $60 \%$ of them satisfy it, more of them satisfy it, computed by the given formula.

\subsection{Weighted Aggregation Functions}

The weighted arithmetic mean defined by

$$
\operatorname{WAM}\left(x_{1}, \cdots, x_{n}\right)=\sum_{i=1}^{n} w_{i} x_{i}
$$

and weighted geometric mean defined by

$$
W G M\left(x_{1}, \cdots, x_{n}\right)=\prod_{i=1}^{n} x_{i}^{w_{i}}
$$

are the most commonly used operators to compute the aggregating of weighted input such that the weighting vector $\mathbf{w}_{(n)}=\left(w_{1}, \cdots, w_{n}\right)$, where $w_{i} \geq 0$ and $\sum_{i=1}^{n} w_{i}=1$, shows the importance degrees of $x_{i} \mathrm{~s}$.

Ordered weighted average (OWA) operator [9,33], which is calculated based on the arithmetic mean, and ordered weighted geometric $(O W G)$ operator [10], which is formulated based on the geometric mean, are other two important aggregation operators for weighted input where the position/order of input has the weight rather than their sources.

The ordered weighted average (OWA) operator is defined by

$$
\operatorname{OWA}\left(x_{1}, \cdots, x_{n}\right)=\sum_{i=1}^{n} w_{i} x_{\sigma(i)}
$$


where $\sigma$ is a permutation on $\{1, \cdots, n\}$ and $x_{\sigma(i)}$ is the $i$ th largest element among $x_{1}, \cdots, x_{n}$. Indeed, OWA opeartor is a revised version of WAM operator, where the reordering step of the OWA operator is carried out to assign the weight $w_{i}$ to the $i$ th place/location not $i$ th value. Similarly the ordered weighted geometric $(O W G)$ operator is defined by

$$
\operatorname{OWG}\left(x_{1}, \cdots, x_{n}\right)=\prod_{i=1}^{n} x_{\sigma(i)} w_{i}
$$

\section{Remark 11. Note that}

- If $w_{1}=1$ and $w_{i}=0$ else, then $O W A:=$ Max and $O W G:=$ Max;

- If $w_{n}=1$ and $w_{i}=0$ else, then $O W A:=$ Min and $O W G:=$ Min;

- If $w_{i}=\frac{1}{n}$, then $O W A:=A M$ and $O W G:=G M$;

- If $n$ is odd, $w_{\frac{n+1}{2}}=1$ and $w_{i}=0$ else, then $O W A:=$ Med and $O W G:=$ Med;

- If $n$ is even, $w_{\frac{n}{2}}=w_{\frac{n}{2}+1}=\frac{1}{2}$, and $w_{i}=0$ else, then OWA $:=$ Med.

According to Remark 11, the ordered weighted averaging (OWA) operator is a useful aggregation technique, which provides a family of aggregation operators including the maximum, the minimum, the median, and the mean value. Especially, the role of $O W A$ weights is very important to determine the type of aggregation to be performed. This has been motivated researchers to extend the OWA operator for a wide range of applications. For instance, Sang and Liu [44] considered the parametric form of a new type of OWA operator, called most preferred ordered weighted average (MP-OWA) operator, to deal with the uncertainty preference information where the weighting vector is determined based on the preferences information between arguments. Liu et al. [45] presented the probabilistic ordered weighted continuous extension of OWA operator. Recently, in [46], Yager proposed a solution to the problem of finding weights when we have a probability distribution over the argument values.

However, the main disadvantage of OWA and OWG operators is ignoring the importance of given arguments $x_{1}, \cdots, x_{n}$ for calculating the aggregated value. That is why they were extended into the induced OWA (or IOWA) operator in [41], and induced OWG (or IOWG) operator in [21], respectively. The IOWA operator, introduced by Yager and Filev [41], and the IOWG operator, given by $\mathrm{Xu}$ and $\mathrm{Da}$ [21], are two important extensions of operators $O W A$ and $O W G$, respectively, to bridge the gap of weighted input arguments that is not mentioned in OWA and $O W G$.

The IOWA operator is defined by

$$
\operatorname{IOW} A\left(\left\langle u_{1}, x_{1}\right\rangle, \cdots,\left\langle u_{n}, x_{n}\right\rangle\right)=\sum_{j=1}^{n} w_{j} y_{j}
$$

and IOWG operator is defined by

$$
\operatorname{IOWG}\left(\left\langle u_{1}, x_{1}\right\rangle, \cdots,\left\langle u_{n}, x_{n}\right\rangle\right)=\prod_{j=1}^{n} y_{j}^{w_{j}}
$$

where $y_{j}$ is the value of $x_{i}$ that has the $j$ th largest $u_{i}$, and $u_{i}$ in $\left\langle u_{i}, x_{i}\right\rangle$ is referred to as the weight of variable $x_{i}$. The weights $w_{1}, \cdots, w_{n}$ such that $\sum_{i=1}^{n} w_{i}=1$ are the associated weights to the IOWA and IOWG operators. Here, the reordering step of $x_{i}$ s is carried out by the variable $u_{i}$ rather than the value of $x_{i}$, using in OWA and OWG. Therefore, the collection $x_{1}, \cdots, x_{n}$ is reordered as $\left\langle\max \left\{u_{i}\right\}, y_{1}\right\rangle \geq \cdots \geq\left\langle\min \left\{u_{i}\right\}, y_{n}\right\rangle$.

However, these extensions have the inherent limitations from OWA operator and OWG operator, concerning the determination of associated weighting vector $\mathbf{w}_{(n)}$ for IOWA and IOWG operators.

More extensions of operators OWA, OWG, IOWA, and IOWG have been discussed to aggregating data with fuzzy and vague information (c.f. $[11,12,15,16,18,20]$ ). 
The weighted minimum and the weighted maximum are also commonly used classes of aggregation operators, discussed first in [47], dealing with objects having non-negative weights $w_{1}, \cdots, w_{n}$ such that $\max _{i=1}^{n} w_{i}=1$. Using the concept of possibility and necessity of fuzzy events, Dubois and Prade [47] proposed the following operators, the so-called the weighted disjunction and weighted conjunction, to interpretation the weighted maximum and weighted minimum,

$$
\operatorname{WMax}\left(x_{1}, \cdots, x_{n}\right)=\max _{i=1}^{n} \min \left(w_{i}, x_{i}\right)
$$

and

$$
\operatorname{WMin}\left(x_{1}, \cdots, x_{n}\right)=\min _{i=1}^{n} \min \left(1-w_{i}, x_{i}\right)
$$

where $\max _{i=1}^{n} w_{i}=1$.

\subsection{Weighted Quasi-Arithmetic Means}

By taking the weighted arithmetic mean $W A M=\sum_{i=1}^{n} w_{i} x_{i}$, where $w_{i} \in[0,1]$ and $\sum_{i=1}^{n} w_{i}=1$, in Equation (4) we get the weighted quasi-arithmetic mean as below.

$$
W A M_{f, w}\left(x_{1}, \cdots, x_{n}\right)=f^{-1}\left(\sum_{i=1}^{n} w_{i} f\left(x_{i}\right)\right)
$$

Obviously, if $f(x)=x$ or $f(x)=\log x$, then we have the weighted arithmetic mean WAM and the weighted geometric mean $W G M$, respectively.

Theorem 4. ([25]) The function $F:[0,1]^{n} \rightarrow \mathbb{R}$ is symmetric, continuous, strictly increasing, idempotent, and bisymmetric if and only if there is a continuous and strictly monotonic function $f:[0,1] \rightarrow \mathbb{R}$ and real numbers $w_{1}, \cdots, w_{n} \in[0,1]$ where $\sum_{i=1}^{n} w_{i}=1$ such that $F:=W A M_{f, w}$ is the weighted quasi-arithmetic mean generated by $f$.

If $p:[0,1] \rightarrow R^{+}$is a positive-valued function, then the $n$-ary function $M:[0,1]^{n} \rightarrow[0,1]$ defined by

$$
M\left(x_{1}, \cdots, x_{n}\right)=\frac{\sum_{i=1}^{n} p\left(x_{i}\right) x_{i}}{\sum_{i=1}^{n} p\left(x_{i}\right)}
$$

is an averaging aggregation function, called mixture operator, that is a generalization of WAM $[48,49]$. However, the monotonicity of mixture operators are not clear. There are some sufficient conditions ensuring the monotonicity of such operators discussed in [48]. If $p: I \rightarrow R^{+}$is a non-decreasing differentiable function, then the next two conditions,

1. $\quad p(x) \geq p^{\prime}(x) \cdot l(I)$ for all $x \in I$ where $l(I)$ is the length of the interval $I$;

2. $p(x) \geq p^{\prime}(x) \cdot(x-\inf I)$ for all $x \in I$.

For example, if $p:[0,1] \rightarrow R^{+}$is given by $p(x)=x+3 / 4$. Then, the function $M:[0,1]^{2} \rightarrow[0,1]$ defined by (14) is an idempotent aggregation function.

By using (13), the quasi-mixture operator generated by $f$ with weight function $p$ is the aggregation function $M_{f, p}:[0,1]^{n} \rightarrow[0,1]$ defined by

$$
M_{f, p}\left(x_{1}, \cdots, x_{n}\right)=f^{-1}\left(\frac{\sum_{i=1}^{n} p\left(x_{i}\right) f\left(x_{i}\right)}{\sum_{i=1}^{n} p\left(x_{i}\right)}\right) .
$$

We can also consider the weighted version of given method in Theorem 3 by using weighted quasi-arithmetic mean formula in (13) as the following. 
Definition 6. Let for any $n \in \mathbb{N}, \mathbf{w}_{(n)}=\left(w_{1}, \cdots, w_{n}\right) \in[0,1]^{n}$ be an $n$-dimensional weighting vector for a list of $n$ arguments $x_{1} \cdots, x_{n}$, such that $\sum_{i=1}^{n} w_{i}=1$.

To determine the relevant weights for all $k$ possible $\alpha$-combinations of $\left\{x_{1} \cdots, x_{n}\right\}$, where $k=\left(\begin{array}{l}n \\ \alpha\end{array}\right)$, the $k \times \alpha$ weighting matrix $U_{\alpha}$ is constructed, where for each $i \in\{1, \cdots, k\}$, the row vector $\mathbf{u}_{i}=\left(u_{i, 1}, \cdots, u_{i, \alpha}\right) \in$ $[0,1]^{\alpha}$ from the matrix $U_{\alpha}$ is defined by

$$
\mathbf{u}_{i}:=\frac{\mathbf{w}_{i}}{\sum_{j=1}^{\alpha} w_{l_{i, \sigma(j)}}}
$$

if $\sum_{j=1}^{\alpha} w_{l_{i, \sigma(j)}} \neq 0$ and else $\mathbf{u}_{i}=\mathbf{0}$ such that $\mathbf{w}_{i}=\left(w_{l_{i, \sigma(1)}}, \cdots, w_{l_{i, \sigma(\alpha)}}\right) \in[0,1]^{\alpha}$ is a subsequence of $\mathbf{w}_{(n)}=\left(w_{1}, \cdots, w_{n}\right)$ corresponding to the ith $\alpha$-combination of $x_{1}, \cdots, x_{n}$.

The next theorem provides a new class of the weighted quasi-arithmetic mean approach so-called $\mathbf{w}$-weighted quasi-arithmetic mean combination operator-based aggregation functions.

Theorem 5. ([38]) Suppose that $\mathbf{w}_{(n)}=\left(w_{1}, \cdots, w_{n}\right) \in \mathbb{I}^{n}$ be an $n$-dimensional rational weighting vector. Let $A, B: \bigcup_{n \in \mathbb{N}} \mathbb{I}^{n} \rightarrow \mathbb{I}$ be (extended) aggregation functions, where $B$ is idempotent and continuous and $A:=W A M_{f}$ by means of a continuous monotone function $f:[0,1] \rightarrow[-\infty,+\infty]$. If $U_{\alpha}$ is the weighting matrix, then function $W F_{\alpha ; B, f}: \bigcup_{n \in \mathbb{N}} \mathbb{I}^{n} \rightarrow \mathbb{I}$ given by

$$
\begin{aligned}
W F_{\alpha ; B, f}^{(n)}\left\langle\left(x_{1}, \cdots, x_{n}\right), \mathbf{w}_{(n)}\right\rangle=B^{(k)}\left(W A M_{f}\left\langle C_{n, \alpha}^{\sigma^{*}(1)}\left\{x_{1}, \cdots, x_{n}\right\}, \mathbf{u}_{1}\right\rangle, \cdots,\right. \\
\\
\left.W A M_{f}\left\langle C_{n, \alpha}^{\sigma^{*}(k)}\left\{x_{1}, \cdots, x_{n}\right\}, \mathbf{u}_{k}\right\rangle\right) ; n \in \mathbb{N}
\end{aligned}
$$

is an idempotent continuous $n$-array function that is called $\mathbf{w}$-weighted quasi arithmetic mean combination operator-based aggregation function.

Example 12. Let $B: \bigcup_{n \in \mathbb{N}}[0,1]^{n} \rightarrow[0,1]$ be the arithmetic mean $A M$.

If $f:=x$ then

$$
W F_{\alpha ; A M, f}\left\langle\left(x_{1}, \cdots, x_{n}\right), \mathbf{w}_{(n)}\right\rangle=\frac{1}{k} \sum_{i=1}^{k} \sum_{j=1}^{\alpha} u_{i, j} \cdot x_{\iota_{i, \sigma(j)}}
$$

If $f:=\log x$ then

$$
W F_{\alpha ; A M, f}\left\langle\left(x_{1}, \cdots, x_{n}\right), \mathbf{w}_{(n)}\right\rangle=\frac{1}{k} \sum_{i=1}^{k} \prod_{j=1}^{\alpha} x_{i_{i, \sigma(j)}} u_{i, j}
$$

where for each $i=1, \cdots, k: u_{i, j}=\frac{w_{t_{i, \sigma(j)}}}{\sum_{j=1}^{\alpha} w_{i, \sigma(j)}}$ and $\sum_{j=1}^{\alpha} w_{t_{i, \sigma(j)}} \neq 0$.

\subsection{Weighted Rule Based on a Convex Combination}

How to modify an unweighted scoring rule to apply for arguments with different importance degrees motivated authors in [39] to present a technique for extending an $n$-ary rule to its weighted version where the proposed method answers the following question.

Problem VII. Constructing aggregation operators $A_{\mathbf{w}} \in \mathcal{A}$ based on a convex combination of unweighted aggregation operators. 
Theorem 6. ([39]) For every unweighted rule, $f$ there exists a weighted rule that is based on $f$, compatible, and locally linear. If $\sigma$ is a permutation that orders the weights as follows, $w_{\sigma(1)} \geq \cdots \geq w_{\sigma(n)}$ and $w_{n+1}=0$, then

$$
f_{w}\left(x_{1}, \cdots, x_{n}\right)=\sum_{i=1}^{n} i \cdot\left(w_{\sigma(i)}-w_{\sigma(i+1)}\right) \cdot f\left(x_{\sigma(1)}, \cdots, x_{\sigma(i)}\right)
$$

is a weighted rule based on $f$.

Then, they proved that if an unweighted rule $f$ is continuous (or monotonic, strictly monotonic, idempotent), then the corresponding weighted rule $f_{w}$ is continuous (monotonic, strictly monotonic, and idempotent) as well. They also showed that $\sum_{i=1}^{n} i \cdot\left(w_{\sigma(i)}-w_{\sigma(i+1)}\right)=\sum_{i=1}^{n} w_{i}=1$ where $w_{\sigma(1)} \geq \cdots \geq w_{\sigma(n)}$ and $w_{n+1}=0$.

Corollary 1. Corresponding to any aggregation function $A$, there exists a weighted aggregation function $A_{w}$ based on the weights $w_{1}, \cdots, w_{n}$ defined by

$$
A_{w}\left(x_{1}, \cdots, x_{n}\right)=\sum_{i=1}^{n} i \cdot\left(w_{\sigma(i)}-w_{\sigma(i+1)}\right) \cdot A\left(x_{\sigma(1)}, \cdots, x_{\sigma(i)}\right)
$$

where $w_{\sigma(1)} \geq \cdots \geq w_{\sigma(n)}$ and $w_{n+1}=0$.

For example, if $A:=\min$ or $A:=\max$ then the weighted minimum and the weighted maximum can be defined as below, respectively.

$$
\min _{w}\left(x_{1}, \cdots, x_{n}\right)=\sum_{i=1}^{n} i \cdot\left(w_{\sigma(i)}-w_{\sigma(i+1)}\right) \cdot \min \left(x_{\sigma(1)}, \cdots, x_{\sigma(i)}\right)
$$

and

$$
\max _{w}\left(x_{1}, \cdots, x_{n}\right)=\sum_{i=1}^{n} i \cdot\left(w_{\sigma(i)}-w_{\sigma(i+1)}\right) \cdot \max \left(x_{\sigma(1)}, \cdots, x_{\sigma(i)}\right)
$$

Remark 12. Using discussed constructing methods in previous sections as the underlying formula, we can provide some more new classes of aggregation operators based on the given technique in (18).

For example, combining formulas given by Equations (7) and (20), we can get a weighted version of Example 11 as below.

$$
F_{\alpha ; \max ^{\prime} \min _{w}}^{(n)}\left(x_{1}, \cdots, x_{n}\right)=\max _{i=1}^{k}\left\{\sum_{k j=1}^{\alpha} j \cdot\left(\mathbf{u}_{\sigma(j)}-\mathbf{u}_{\sigma(j+1)}\right) \cdot \min \left(x_{\sigma(1)}, \cdots, x_{\sigma(j)}\right)\right\}
$$

Example 13. Reconsider Example 11 where $B:=\max$ and $A:=\min _{w}$. Take the weighting vector $\mathbf{w}_{(4)}=$ $\left(w_{1}, w_{2}, w_{3}, w_{4}\right)$ such that $w_{i} \in[0,1], \sum_{i=1}^{4} w_{i}=1, w_{1} \geq w_{2} \geq w_{3} \geq w_{4}$ and $w_{5}=0$. Then, using (22), the aggregation operator $F_{3, \max _{\text {, }} \min _{w}}^{(4)}:[0,1]^{4} \rightarrow[0,1]$ can be defined as below. 


$$
\begin{aligned}
& F_{3 ; \max _{\text {min }} w}^{(4)}\left(x_{1}, x_{2}, x_{3}, x_{4}\right)=\max \left(\left(\frac{w_{1}}{\sum_{i=1}^{3} w_{i}}-\frac{w_{2}}{\sum_{i=1}^{3} w_{i}}\right) x_{1}+2\left(\frac{w_{2}}{\sum_{i=1}^{3} w_{i}}-\frac{w_{3}}{\sum_{i=1}^{3} w_{i}}\right) \min \left\{x_{1}, x_{2}\right\}\right. \\
& +3 \frac{w_{3}}{\sum_{i=1}^{3} w_{i}} \min \left\{x_{1}, x_{2}, x_{3}\right\},\left(\frac{w_{1}}{\sum_{i=1, i \neq 3}^{4} w_{i}}-\frac{w_{2}}{\sum_{i=1, i \neq 3}^{4} w_{i}}\right) x_{1}+2\left(\frac{w_{2}}{\sum_{i=1, i \neq 3}^{4} w_{i}}-\frac{w_{4}}{\sum_{i=1, i \neq 3}^{4} w_{i}}\right) \min \left\{x_{1}, x_{2}\right\} \\
& +3 \frac{w_{4}}{\sum_{i=1, i \neq 3}^{4} w_{i}} \min \left\{x_{1}, x_{2}, x_{4}\right\},\left(\frac{w_{1}}{\sum_{i=1, i \neq 2}^{4} w_{i}}-\frac{w_{3}}{\sum_{i=1, i \neq 2}^{4} w_{i}}\right) x_{1}+2\left(\frac{w_{3}}{\sum_{i=1, i \neq 2}^{4} w_{i}}-\frac{w_{4}}{\sum_{i=1, i \neq 2}^{4} w_{i}}\right) \min \left\{x_{1}, x_{3}\right\} \\
& \quad+3 \frac{w_{4}}{\sum_{i=1, i \neq 2}^{4} w_{i}} \min \left\{x_{1}, x_{3}, x_{4}\right\},\left(\frac{w_{2}}{\sum_{i=2}^{4} w_{i}}-\frac{w_{3}}{\sum_{i=2}^{4} w_{i}}\right) x_{2}+2\left(\frac{w_{3}}{\sum_{i=2}^{4} w_{i}}-\frac{w_{4}}{\sum_{i=2}^{4} w_{i}}\right) \min \left\{x_{2}, x_{3}\right\} \\
& \left.+3 \frac{w_{4}}{\sum_{i=2}^{4} w_{i}} \min \left\{x_{2}, x_{3}, x_{4}\right\}\right)
\end{aligned}
$$

\section{Discussion}

This study presents an overview of three constructing methods to aggregation functions, namely, transformation, composition, and convex combination, and their applications in group decision-making problems.

The following example compares the effect of choosing different aggregation methods on final result of aggregating data in a decision-making problem.

Example 14. (Student evaluation based on the academic report) To evaluate the students' academic performance based on their final courses' marks where all courses have the same importance degree the mean value operator AM can be applied simply.

Let us suppose that four students are evaluated based on their final marks, that are calculated from 0 to 10, in three different courses as shown in Table 1 where their marks are divided by 10.

Table 1. Evaluation of students by AM.

\begin{tabular}{lcccc}
\hline & Course 1 & Course 2 & Course 3 & AM \\
\hline Student 1 & 0.9 & 0.5 & 0.7 & 0.7 \\
Student 2 & 0.7 & 0.7 & 0.7 & 0.7 \\
Student 3 & 0.5 & 0.6 & 0.4 & 0.5 \\
Student 4 & 0.85 & 0.7 & 0.5 & 0.68 \\
\hline
\end{tabular}

According to Table 1, Student 1 and Student 2 have the best performance among all 4 students. As AM is a self-dual aggregation function, to find out the degree of failure of each student we only need to compute the $1-A M$. This means that student 3 by the highest failure degree 0.5 has the worst performance.

Now let these courses have different weights as $\mathbf{w}_{(3)}=(0.3,0.5,0.2)$. Therefore to compare the achievement of students we must use the WAM operator rather that AM.

By Table 2, it is easy to see that in this case Student 4 has better performance.

Table 2. Evaluation of students by WAM.

\begin{tabular}{lcccc}
\hline & $\begin{array}{c}\text { Course 1 } \\
\mathbf{0 . 3}\end{array}$ & $\begin{array}{c}\text { Course 2 } \\
\mathbf{0 . 5}\end{array}$ & $\begin{array}{c}\text { Course 3 } \\
\mathbf{0 . 2}\end{array}$ & WAM \\
\hline Student 1 & 0.9 & 0.5 & 0.7 & 0.66 \\
Student 2 & 0.7 & 0.7 & 0.7 & 0.7 \\
Student 3 & 0.5 & 0.6 & 0.4 & 0.53 \\
Student 4 & 0.85 & 0.7 & 0.5 & 0.705 \\
\hline
\end{tabular}

In the next step, suppose that the correlation between students' achievement and examination timetable is being studied where the weighting vector $\mathbf{w}_{(3)}=(0.3,0.5,0.2)$ has been devoted based on the courses' exam date in the examination schedule. 
As shown in Table 3, in this case, Student 1 has the best performance during the examination week based on aggregating operator IOWA.

Table 3. Evaluation of students by IOWA.

\begin{tabular}{lcccc}
\hline & $\begin{array}{c}\text { Course 1 } \\
\text { First Exam } \\
\mathbf{0 . 3}\end{array}$ & $\begin{array}{c}\text { Course 2 } \\
\text { Third Exam } \\
\mathbf{0 . 2}\end{array}$ & $\begin{array}{c}\text { Course 3 } \\
\text { Second Exam } \\
\mathbf{0 . 5}\end{array}$ & IOWA \\
\hline Student 1 & 0.9 & 0.5 & 0.7 & 0.72 \\
Student 2 & 0.7 & 0.7 & 0.7 & 0.7 \\
Student 3 & 0.5 & 0.6 & 0.4 & 0.47 \\
Student 4 & 0.85 & 0.7 & 0.5 & 0.645 \\
\hline
\end{tabular}

Let us now consider more challenging case where only 2 courses of these three courses are enough to evaluate the performance of students. In this case, we use the hybrid operator $F_{2 ; M a x, W A M}^{(3)}$ to find the overall score of each student.

Thus according to Table 4, Student 1 has the best record during the examination week.

Table 4. Evaluation of students by $F_{2 ; M a x, W A M}^{(3)}$.

\begin{tabular}{lcccc}
\hline & Course 1 & Course 2 & Course 3 & $\boldsymbol{F}_{\mathbf{2} \text {;Max,WAM }}^{(3)}$ \\
& $\mathbf{0 . 3}$ & $\mathbf{0 . 5}$ & $\mathbf{0 . 2}$ & 0.82 \\
Student 1 & 0.9 & 0.5 & 0.7 & 0.7 \\
Student 2 & 0.7 & 0.7 & 0.7 & 0.56 \\
Student 3 & 0.5 & 0.6 & 0.4 & 0.75 \\
Student 4 & 0.85 & 0.7 & 0.5 & \\
\hline
\end{tabular}

\section{Conclusions}

In decision situations, aggregating for reaching to consensus is one of the most important steps before taking the final action. This means that the used aggregating method affects the final solution. Different aggregating techniques have been discussed in literature; however, the most useful one is aggregation functions. In this paper, we have recalled properties and classification of aggregation functions. Some construction methods to generate new aggregation operators based on the existing ones have been also reviewed. Based on their characteristics, these techniques are classified into the following three groups; (1) transformation (which can produce linear transformation and dual of aggregation functions), (2) composition (that can cope with the problem of multi-source data), and (3) weighted rule of aggregation operators based on their convex combination (which permits data with different importance degrees). Especially, we have focused on the applications and consensus problems that may be handled by each of these methods. We also briefly discussed the differences between them by a comparative example.

Moreover, to develop new aggregation operators in future research, this overview gives an insight to the researchers. However, it is observed that, to date, many efforts have been made to develop aggregation functions theory, there are some gaps that need to be discussed by researchers in the future. For instance, there exist situations with multi-source data where some of the input information is dynamic or not complete that means the aggregating progress over each source can be dynamic or partial (such as negotiation process in management level). Composition of aggregation functions over different sub-groups of data where there are some incomplete/missing information may be the solution that can be discussed in another paper.

Author Contributions: Conceptualization, A.Z.K.; methodology, A.Z.K.; writing-original draft preparation, A.Z.K.; writing-review and editing, A.Z.K. and A.K.; project administration, A.K. All authors have read and agreed to the published version of the manuscript. 
Funding: This research was supported by the Fundamental Research Grant Schemes, Reference No: FRGS/1/2018/STG06/UPM/01/3 and Ref. NO.: FRGS/1/2019/STG06/UPM/02/6, awarded by the Malaysia Ministry of Higher Education.

Conflicts of Interest: The authors declare no conflict of interest.

\section{References}

1. Herrera-Viedma, E.; Cabrerizo, F.J.; Kacprzyk, J.; Pedrycz, W. A review of soft consensus models in a fuzzy environment. Inf. Fusion 2014, 17, 4-13. [CrossRef]

2. Xu, G.1.; Wan, S.P.; Wang, F.; Dong, J.V.; Zeng, Y.F. Mathematical programming methods for consistency and consensus in group decision-making with intuitionistic fuzzy preference relations. Knowl.-Based Syst. 2016, 98, 30-43. [CrossRef]

3. Zahedi Khameneh, A.; Kilicman, A. Multi-attribute decision-making based on soft set theory: A systematic review. Soft Comput. 2019, 23, 6899-6920. [CrossRef]

4. De Baets, B.; Mesiar, R. Triangular norms on product lattices. Fuzzy Sets Syst. 1999, 104, 61-75. [CrossRef]

5. Choquet, G. Theory of capacities. Ann. Inst. Fourier. 1953, 5, 131-295. [CrossRef]

6. Sugeno, M. Theory of Fuzzy Integral and Its Application. Ph.D. Thesis, Tokyo Institute of Technology, Tokyo, Japan, 1974.

7. Zadeh, L.A. Fuzzy sets. Inf. Comput. 1965, 8, 338-353. [CrossRef]

8. Molodtsov, D. Soft set theory-first results. Comput. Math. Appl. 1999, 37, 19-31. [CrossRef]

9. Yager, R.R. On ordered weighted averaging aggregation operators in multicriteria decisionmaking. In Readings in Fuzzy Sets for Intelligent Systems; Elsevier: New York, NY, USA, 1993; pp. 80-87.

10. Chiclana, F.; Herrera, F.; Herrera-Viedma, E. The Ordered Weighted Geometric Operator: Properties and Application in MCDM Problems. In Proceedings of the 8th Conference on Information Processing and Management of Uncertainty in Knowledge Based Systems (IPMU), Madrid, Spain, 15-19 July 2002; pp. $985-991$.

11. Xu, Z.S.; Yager, R.R. Some geometric aggregation operators based on intuitionistic fuzzy sets. Int. J. Gen. Syst. 2006, 35, 417-433. [CrossRef]

12. Xu, Z.S. Intuitionistic fuzzy aggregation operators. IEEE Trans. Fuzzy Syst. 2007, 15, 1179-1187.

13. $\mathrm{Xu}, \mathrm{Z} . S . ;$ Chen, J. On geometric aggregation over interval-valued intuitionistic fuzzy information. In Proceedings of the 4th International Conference on Fuzzy Systems and Knowledge Discovery (FSKD), Haikou, China, 24-27 August 2007; pp. 466-471.

14. Wang, J.Q.; Zhang, Z. Multi-criteria decision-making method with incomplete certain information based on intuitionistic fuzzy number. Control Decis. 2009, 24, 226-230.

15. Wei, G. Some induced geometric aggregation operators with intuitionistic fuzzy information and their application to group decision-making. Appl. Soft Comput. 2010, 10, 423-431. [CrossRef]

16. Zhao, H.; Xu, Z.S.; Ni, M.F.; Liu, S.S. Generalized aggregation operators for intuionistic fuzzy sets. Int. J. Intell. Syst. 2010, 25, 1-30. [CrossRef]

17. Merigo, J.M.; Gil-Lafuente, A.M. Fuzzy induced generalized aggregation operators and its application in multi-person decision-making. Expert Syst. Appl. 2011, 38, 9761-9772. [CrossRef]

18. $\mathrm{Xu}, \mathrm{Y}$.; Wang, H. The induced generalized aggregation operators for intuitionistic fuzzy sets and their application in group decision-making. Appl. Soft Comput. 2012, 12, 1168-1179. [CrossRef]

19. Shakeel, M.; Abdullah, S.; Sajjad Ali Khan, M.; Rahman, K. Averaging aggregation operators with interval pythagorean trapezoidal fuzzy numbers and their application to group decision-making. Punjab Univ. J. Math. 2018, 50, 147-170.

20. Zahedi Khameneh, A.; Kilicman, A. m-polar fuzzy soft weighted aggregation operators and their applications in group decision-making. Symmetry 2018, 10, 636. [CrossRef]

21. Xu, Z.S.; Da, Q.L. An Overview of operators for aggregating information. Int. J. Intell. Syst. 2003, 18, 953-969. [CrossRef]

22. Calvo, T.; Kolesárová, A.; Komorníková, M.; Mesiar, R. Aggregation operators: Properties, classes and construction methods. In Aggregation Operators. Studies in Fuzziness and Soft Computing; Calvo, T., Mayor, G., Mesiar, R., Eds.; Physica: Heidelberg, Germany, 2002; Volume 97, pp. 3-104.

23. Grabisch, M.; Marichal, J.L.; Mesiar, R.; Pap, E. Aggregation functions: Construction methods, conjunctive, disjunctive and mixed classes. Inf. Sci. 2011, 181, 23-43. [CrossRef] 
24. Martinez, D.L.L.R.; Acosta, J.C. Aggregation operators review-Mathematical properties and behavioral measures. Int. J. Intell. Syst. Tech. Appl. 2015, 10, 63-76. [CrossRef]

25. Grabisch, M.; Marichal, J.L.; Mesiar, R.; Pap, E. Aggregation functions: Means. Inf. Sci. 2011, 181, 1-22. [CrossRef]

26. Rosanisah, M.; Abdullah, L. Aggregation methods in group decision-making: A decade survey. Informatica 2017, 41, 71-86.

27. Calvo, T.; Pradera, A. Double aggregation operators. Fuzzy Sets Syst. 2004, 142, 15-33. [CrossRef]

28. García-Lapresta, J.L.; Pereira, R.A.M. The self-dual core and the anti-self-dual remainder of an aggregation operator. Fuzzy Sets Syst. 2008, 159, 47-62. [CrossRef]

29. Aczél, J. On mean values. Bull. Am. Math. Soc. 1984, 54, 392-400. [CrossRef]

30. Mesiar, R.; Špirkova, J.; Vavrıkova, L. Weighted aggregation operators based on minimization. Inf. Sci. 2008, 178, 1133-1140. [CrossRef]

31. Špirková, J.; Beliakov, G.; Bustince, H.; Fernandez, J. Mixture functions and their monotonicity. Inf. Sci. 2019, 481, 520-549. [CrossRef]

32. Del Moral, M.J.; Chiclana, F.; Tapia, J.M.; Herrera-Viedma, E. A comparative study on consensus measures in group decision-making. Int. J. Intell. Syst. 2018, 33, 1624-1638. [CrossRef]

33. Bordogna, G.; Fedrizzi, M.; Pasi, G. A linguistic modeling of consensus in group decision-making based on OWA operators. IEEE Trans. Syst. Man. Cybern. A 1997, 27, 126-133. [CrossRef]

34. Yager, R.; Rybalov, A. Bipolar aggregation using the Uninorms. Fuzzy Optim. Decis. Mak. 2011, 10, 59-70. [CrossRef]

35. Dubois, D.; Prade, H. On the use of aggregation operations in information fusion processes. Fuzzy Sets Syst. 2004, 142, 143-161. [CrossRef]

36. Komornikova, M.; Mesiar, R. Aggregation functions on bounded partially ordered sets and their classification. Fuzzy Sets Syst. 2011, 175, 48-56. [CrossRef]

37. Cauchy, A.L. Cours D'analyse de L'Ecole Royale Polytechnique. In Analyse Algebrique; Debure: Paris, France, 1821; Volume I.

38. Zahedi Khameneh, A.; Kilicman, A. A fuzzy majority-based construction method for composed aggregation functions by using combination operator. Inf. Sci. 2019, 505, 367-387. [CrossRef]

39. Fagin, R.; Wimmers, E.L. A formula for incorporating weights into scoring rules. Theor. Comput. Sci. 2000, 239, 309-338. [CrossRef]

40. Yager R.R. A new methodology for ordinal multiobjective decisions based on fuzzy sets. In Readings in Fuzzy Sets for Intelligent Systems; Elsevier: New York, NY, USA, 1993; pp. 751-756.

41. Yager, R.R.; Filev, D.P. Induced ordered weighted averaging operators. IEEE Trans. Syst. Man. Cybern. B Cybern. 1999, 29, 141-150. [CrossRef] [PubMed]

42. Kacprzyk, J. Group decision-making with a fuzzy linguistic majority. Fuzzy Sets Syst. 1986, 18, 105-118. [CrossRef]

43. Kacprzyk, J.; Fedrizzi, M.; Nurmi, H. Group decision-making and consensus under fuzzy preferences and fuzzy majority. Fuzzy Sets Syst. 1992, 49, 21-31. [CrossRef]

44. Sang, X.; Liu, X. Parametric extension of the most preferred OWA operator and its application in search engine's rank. J. Appl. Math. 2013. [CrossRef]

45. Liu, X.; Han, B.; Chen, H.; Zhou, L. The probabilistic ordered weighted continuous OWA operator and its application in group decision-making. Int. J. Mach. Learn. Cybern. 2019, 10, 705-715. [CrossRef]

46. Yager, R.R. OWA aggregation with an uncertainty over the arguments. Inf. Sci. 2019, 52, 206-212. [CrossRef]

47. Dubois, D.; Prade, H. Weighted minimum and maximum operations in fuzzy set Theory. Inf. Sci. 1986, 39, 205-210. [CrossRef]

48. Ribeiro, R.A.; Pereira, R.A.M. Weights as functions of attribute satisfaction values. In Proceedings of the Workshop on Preference Modelling and Applications (EUROFUSE), Granada, Spain, 25-27 April 2001.

49. Ribeiro, R.A.; Pereira, R.A.M. Generalized mixture operators using weighting functions: A comparative study with WA and OWA. Eur. J. Oper. Res. 2003, 145, 329-342. [CrossRef]

(C) 2020 by the authors. Licensee MDPI, Basel, Switzerland. This article is an open access article distributed under the terms and conditions of the Creative Commons Attribution (CC BY) license (http:/ / creativecommons.org/licenses/by/4.0/). 